INPLASY

PROTOCOL

To cite: Ren et al. Efficacy of Kinesio Tape in reducing facial swelling, pain and trismus after mandibular third molar surgery: A systematic review and metaanalysis. Inplasy protocol

202180069. doi:

10.37766/inplasy2021.8.0069

Received: 18 August 2021

Published: 18 August 2021

Corresponding author:

Xiaochun Ren

renxiaochun@hospital.cqmu.edu.cn

Author Affiliation:

Stomatological Hospital of Chongqing Medical University

Support: Chinese NFSC (31871464).

Review Stage at time of this submission: Preliminary searches.

Conflicts of interest:

None declared.

\section{Efficacy of Kinesio Tape in reducing facial swelling, pain and trismus after mandibular third molar surgery: A systematic review and meta-analysis}

Ren, XC1; Qin, HY2; Huang, S3; Ji, P4.

Review question / Objective: The aim of this systematic review is to evaluate the efficacy of Kinesio Tape in reducing postoperative morbidity (swelling, pain and trismus) after mandibular third molar surgery. To this end, the proposed systematic review will address the following question: Can the application of Kinesio Tape reduce the postoperative facial swelling, pain and trismus after mandibular third molar surgery?

Condition being studied: The mandibular third molar surgery generally results in varying degrees of postoperative morbidity such as swelling, pain and trismus. Application of Kinesio Tape (KT) has become increasingly popular in various clinical cases for its improvement of postoperative pain and swelling, and several studies have reported its application in the mandibular third molar surgery.

INPLASY registration number: This protocol was registered with the International Platform of Registered Systematic Review and Meta-Analysis Protocols (INPLASY) on 18 August 2021 and was last updated on 18 August 2021 (registration number INPLASY202180069).

\section{INTRODUCTION}

Review question / Objective: The aim of this systematic review is to evaluate the efficacy of Kinesio Tape in reducing postoperative morbidity (swelling, pain and trismus) after mandibular third molar surgery. To this end, the proposed systematic review will address the following question: Can the application of Kinesio Tape reduce the postoperative facial swelling, pain and trismus after mandibular third molar surgery?

Condition being studied: The mandibular third molar surgery generally results in varying degrees of postoperative morbidity such as swelling, pain and trismus. 
Application of Kinesio Tape (KT) has become increasingly popular in various clinical cases for its improvement of postoperative pain and swelling, and several studies have reported its application in the mandibular third molar surgery.

\section{METHODS}

Search strategy: A comprehensive literature search in PubMed, Embase, Web of Science, CENTRAL (Cochrane Central Register of Controlled Trials), and Scopus was conducted to identify papers published up to June 2021 without language restriction. A combination of medical subject heading (MeSH) terms and free terms was used for the search. In addition, A search of the grey literature (Google Scholar, ClinicalTrials.gov and Open Grey) and the references lists of the selected papers was performed in order to identify any reference that had not been found in the search strategy and could be included. The main search strategy of each database was as follows: 1) PubMed (((((Third molar[Title/Abstract]) OR (Third Molars[Title/Abstract])) OR (Wisdom Tooth[Title/Abstract])) OR (Wisdom Teeth[Title/Abstract])) OR ("Molar, Third"[Mesh])) AND $((()(()(()((T a p e$, Athletic[Title/Abstract]) OR (Orthotic Tape[Title/Abstract])) OR (Tape, Orthotic[Title/Abstract])) OR (Kinesio Tape[Title/Abstract])) OR (Kinesio Tapes[Title/Abstract])) OR (Tape, Kinesio[Title/Abstract])) OR (Tapes, Kinesio[Title/Abstract])) OR (Kinesiotape[Title/Abstract])) OR (Kinesio[Title/Abstract])) OR (Kinesiotaping[Title/Abstract])) OR (kinesiologic[Title/Abstract])) OR (kinesiology[Title/Abstract])) OR ("Athletic Tape"[Mesh])). 2) Embase ('third molar'/exp OR 'third molar' OR 'third molar':ab,ti OR 'wisdom tooth':ab,ti OR 'wisdom teeth':ab,ti OR 'dens serotinus':ab,ti OR 'dentes serotini':ab,ti) AND ('athletic tape'/ exp OR 'athletic tape' OR kinesiotherapy:ab,ti OR kinesio:ab,ti OR kinesiotape:ab,ti OR kinesiologic:ab,ti OR kinesiology:ab,ti). 3) Web of Science (TS=("third molar")) OR TS=("wisdom tooth") AND TS=(kinesio*). 4) CENTRAL (MeSH descriptor: [Molar, Third] explode all trees) AND (MeSH descriptor: [Athletic Tape] explode all trees). 5) Scopus (KEY ( "third molar" ) OR KEY ( "wisdom tooth " )) AND (KEY (kinesio tape) OR KEY (kinesiology)).

Participant or population: Healthy patients with impacted mandibular third molars justifying surgical extraction will be included in this review, with no restriction of age, gender and ethnicity. The exclusion criteria are systemic diseases, pathologic conditions in the third molar region, pregnant or lactating women.

Intervention: Application of Kinesio Tape after mandibular third molar surgery to reduce postoperative swelling, pain and trismus.

Comparator: Placebo taping or no taping after mandibular third molar surgery.

Study designs to be included: We will include only randomized controlled trials (RCTs).

Eligibility criteria: Studies will be selected according to the PICOS criteria (Participant, intervention, comparator, outcomes, and study design) outlined in the referred sections. When a multi-arm study compares the efficacy of Kinesio Tape with other treatment modalities, only the data come from the Kinesio Tape group and control group will be included. No restriction of country, publications status, setting, or language will be applied.

Information sources: We searched the following databases: PubMed, Embase, Web of Science, CENTRAL (Cochrane Central Register of Controlled Trials), and Scopus. We used the PICOS strategy for the research question construction and evidence search. In addition, A search of the grey literature (Google Scholar, ClinicalTrials.gov and Open Grey) and the references lists of the selected papers was performed in order to identify any reference that had not been found in the search strategy and could be included. 
Main outcome(s): 1) Pain: postoperative pain in the first week evaluated by visual analog scales (VAS). 2) Swelling: facial measurement between preset facial marking points in the first week. 3 ) Trismus: the distance between the incisal edges of the upper and lower central incisors in the first week.

\section{Additional outcome(s): None.}

Data management: All relevant studies will be imported into Endnote X9 software where duplicates will be automatically removed. The titles and abstracts of these studies will be independently screened by two reviewers based on the inclusion criteria and prespecified PICOS framework. Then, the selected studies will be read in full to confirm their eligibility. Any disagreement will be resolved by discussion with a third reviewer. Data extraction will be completed using a predefined and standardized Microsoft Excel form, including the information of author, publication time, study design, details of the interventions, characteristics of participants, follow up period, and outcome measures. The subsequent analyses will be performed using Review Manager (RevMan) version 5.3.

Quality assessment / Risk of bias analysis: Two reviewers will independently assess the risk of bias of the included studies according to the guidelines recommended by the Cochrane Collaboration, and disagreements will be resolved through discussion. The criteria include sequence generation, allocation concealment, blinding, incomplete outcome data, selective outcome reporting, and other sources of bias. The quality of evidence will be assessed using the Grading of Recommendations Assessment, Development, and Evaluation (GRADE) system.

Strategy of data synthesis: Statistical analyses will be performed using RevMan 5.3. Heterogeneity will be evaluated by a test for heterogeneity (12 statistic) on the level of $a=0.10$. If there is substantial or considerable heterogeneity $(12>50 \%)$, the results will be assessed by random-effects model; if not, fixed-effects model will be used. Since the main outcomes (swelling, pain and trismus) are presented as continuous data, the standardized mean difference (SMD) or weighted mean difference (WMD) will be calculated as effect measures. In cases where the outcome measures are numerically similar and are in the same unit WMD will be measured otherwise SMD will be measured. The statistical significance for the hypothesis test will be set at $a=0.05$ (two-tailed z-tests). Publication bias will be assessed by drawing funnel plot if the number of included studies exceed 10.

Subgroup analysis: Where possible, we plan to performed subgroup analysis in relation to different follow-up period. Any other subset that may arise will be also analyzed.

Sensitivity analysis: None.

Language: There are no language restrictions.

Country(ies) involved: China.

Keywords: kinesio tape, third molar, pain, swelling, trismus, systematic review.

Dissemination plans: The systematic review will be submitted to a peer-reviewed journal for publication.

Contributions of each author:

Author 1 - Xiaochun Ren.

Author 2 - Shun Huang.

Author 3 - Haoyang Qin.

Author 4 - Ping Ji. 